\title{
Influencia del riempo de almacenamiento sobre la dureza y rugosidad de materiales para rebasados resilentes
}

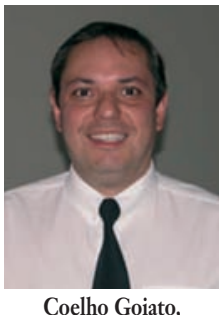

Coelho Goiato, Marcelo

\author{
Influence of storage time on the hardness and roughness of soft liners materials
}

Coelo Goiato, Marcelo*
Micheline Dos Santos, Daniela**
Falcón Antenucci, Rosse Marlu**
Do Prado Ribeiro, Paulaa*

* Profesores del Departamento de Materiales Odontológicos y Prótesis de la Facultad de Odontología de Araçatuba-UNESP

** Doctorado del Curso de pos-graduación de Odontología-UNESP

\section{Correspondencia}

Marcelo Coelho Goiato

Facultad de Odontología de Araçatuba. UNESP.

Departamento de Materiales y Prótesis. Rua José Bonifácio, 1193-Vila Mendoça Araçatuba-Sao Paulo-Brasil

CEP:16015-050.

E-mail: goiato@foa.unesp.br
Resumen: Fundamento: Los materiales para rebasados resilentes de prótesis, presentan ciertas características clínicamente importantes que pueden alterarse con el tiempo de uso, entre ellas la dureza, y rugosidad. El objetivo de este estudio fue evaluar la influencia del almacenamiento sobre la dureza Shore A y rugosidad de cuatro materiales resilentes para rebasado. Materiales y método: Fueron utilizados 4 materiales resilentes: Dentuflex, Coe-Soft, Dentusoft y Dinabase, 3 temporales y un permanente; para cada material fueron confeccionadas 7 muestras. Se evaluó la dureza y rugosidad inicialmente, a los 30 y 90 días de almacenamiento en agua destilada a $35 \pm 2^{\circ} \mathrm{C}$. Los resultados fueron sometidos al análisis de varianza (ANOVA) y las medias comparadas por el Test de Tukey $(p<0,05)$. Resultados: Dentuflex, Coe-Soft, Dentusoft y Dinabase presentaron los mayores valores medios iniciales de dureza, en este orden. Con respecto a la rugosidad, Dentuflex, Dentusoft, Coe-Soft y Dinabase presentaron los mayores valores medios iniciales. Después del periodo de almacenamiento se observó un aumento significativo de los valores de dureza en todos los materiales. El material Dentuflex presentó valores de rugosidad significativamente menores, mientras que los valores de los otros materiales aumentaron significativamente. Conclusión: El tiempo de almacenamiento influenció sobre la dureza y rugosidad todos los materiales.

Palabras clave: Materiales resilentes. Dureza. Rugosidad.

Abstract: Statement of problem: Resilient soft liners materials have many important features that could be altered over time, like hardness and roughness. The aim of this study was to assess the influence of water storage on the hardness Shore A and roughness of four resilient soft liners materials. Materials and methods: Four materials were used: Dentuflex, Coe-Soft, Dentusoft and Dinabase, three temporary and one permanent material. For each material 7 specimens were made. The hardness and the roughness were evaluated in the initial period, after 30 and 90 days of distilled water storage at $35 \pm 2{ }^{\circ} \mathrm{C}$. The results were submitted to analyses of variance (ANOVA) and the averages compared by Tukey's Test $(p<0.05)$. Results: Dentuflex, Coe-Soft, Dentusoft and Dinabase displayed higher initial average of hardness values, whereas Dentuflex, Dentusoft, Coe-Soft and Dinabase higher initial average of roughness values. After water storage a significant increase in the hardness values in all materials was observed. Dentuflex material showed smaller roughness values, while the other materials increased significantly. Conclusion: Time storage influenced all the materials analyzed with respect to hardness and roughness.

Key words: Soft liners materials, Hardness, Roughness.

BIBLID [1138-123X (2007)12:1-2; enero-junio 1-104]

Coelho Goiato M, Micheline Dos Santos D, Falcón Antenucci RM, Do Prado Ribeiro P. Influencia del tiempo de almacenamiento sobre la dureza y rugosidad de materiales para rebasados resilentes. RCOE 2007;12(1-2):67-72. 


\section{Introducción}

Los pacientes desdentados totales - parciales que utilizan dentadura durante mucho tiempo son propensos a una excesiva reabsorción del hueso alveolar y lesiones localizadas en la mucosa alveolar. Para resolver estos problemas son frecuentemente utilizados los materiales blandos y flexibles resilientes con el propósito de disminuir la tensión, proporcionar una presión masticatoria equilibrada en los tejidos de soporte y aumentar la retención de la dentadura'. Estos materiales son adecuados para tratamiento de pacientes con reabsorción ósea, atrofia del reborde residual, mucosa relativamente fina y no resiliente, reborde de tipo «afilado», bruxismo, xerostomía, y cuando las dentaduras se oponen a dentición natu$\mathrm{ral}^{2-4}$.

Los materiales resilientes son clasificados básicamente en dos grupos: materiales a base de elastómeros de silicona y a base de resina acrílica ${ }^{5,6}$. Los materiales a base de elastómeros de silicona presentan en su composición polímero de dimetil siloxano ${ }^{7}$, y los materiales a base de resina acrílica están compuestos por polvo conteniendo polímeros y copolímeros, además de un líquido conteniendo monómero acrílico y plastificante. 8 Algunos materiales para rebasados resilientes a base de resina acrílica no presentan ligaciones cruzadas después de la polimerización. Estos son denominados acondicionadores de tejidos, que se diferencian de los materiales para rebasado por el hecho de ser materiales de uso temporal ${ }^{9}$.

La concentración de plastificante en el líquido influye directamente en el grado de flexibilidad de la base resiliente. Así, cuanto menor o mayor la cantidad de plastificante, menor o mayor será el grado de dureza del material ${ }^{7}$. Por lo tanto, la característica de resilencia de estos materiales es conferida por la presencia de este componente. Con el paso del tiempo, los plastificantes sufren un proceso llamado lixiviación por el cual pierden sus constituyentes solubles ocasionando aumento de la dureza ${ }^{10}$ y de la rugosidad superficial, afectando directamente a la longevidad del material ${ }^{11}$.

La eficacia de los materiales para rebasado blandos es atribuida a sus propiedades visco-elásticas que están relacionadas a la flexibilidad del material ${ }^{12-17}$. Se sabe que, cuanto menor la dureza de estos materiales mayor la capacidad de distribuir y absorber la energía generada por las fuerzas oclusales durante la función de la prótesis.

De acuerdo con Khaled et al. ${ }^{18}$, la rugosidad superficial es un factor significativo en la fijación y retención de microorganismos. Esta es diferente entre los diversos materiales para rebasado resilientes ${ }^{19}$. En ese sentido, los microorganismos de la placa pueden adherirse a la superficie de la dentadura exponiendo al paciente a una infección.

El propósito de este estudio fue evaluar la influencia del tiempo de almacenamiento sobre la dureza Shore A y rugosidad superficial de materiales para rebasados resilientes.

\section{Materiales y Métodos}

Esta investigación es un estudio de tipo comparativo y fue realizado en el laboratorio de investigación del Departamento de Materiales Odontológicos y Prótesis de la Facultad de Odontología de Araçatuba - Universidad Estadual Paulista.

\section{Materiales}

Los materiales utilizados fueron 4 resinas resilientes autopolimerizables para rebasado de dentaduras: CoeSoft, Dentusoft, Dinabase (temporales) y Dentuflex (permanente).

En la tabla 1 se muestran los materiales utilizados en este estudio, su tipo, marca, fabricante, país y su composición química.

\section{Método}

Para cada material fueron obtenidas 10 muestras confeccionadas por medio de matrices de silicona Zetalabor (Zhermack, Rovigo, Italia) de 30 $\mathrm{mm}$ de diámetro, $1 \mathrm{~mm}$ de espesor en los bordes y $8 \mathrm{~mm}$ en el centro. Estas matrices fueron incluidas en muflas plásticas para microondas (Onda Cryl, Artigos Odontológicos Clássico Ltda, São Paulo, Brasil) utilizando yeso especial tipo IV (Vel-mix, Kerr Lab, EUA). Después del fraguado del yeso las muflas fueron abiertas y los discos eliminados.

Los materiales para rebasado de dentaduras (Coe Soft, Dentusoft, Dentuflex y Dinabase) fueron proporcionados y manipulados de acuerdo con las instrucciones del fabricante. Después de la manipulación, éstos fueron acondicionados en los moldes contenidos en las muflas y comprimidos en una prensa hidráulica (Midas Dental Products Ltda., São Paulo, Brasil) con una fuerza de 9800 Newtons. Después de la polimerización las muestras fueron extraídas y los bor- 


\begin{tabular}{|c|c|c|c|}
\hline Material & Marca & Fabricante & Composición \\
\hline $\begin{array}{l}\text { Material para rebasado resiliente } \\
\text { temporal a base de resina acrílica } \\
\text { (autocurado) }\end{array}$ & Coe-Soft ${ }^{\circledR}$ & América Inc. Alsip, EUA & $\begin{array}{l}\text { Líquido: Di-n-Butil ftalato, Alcohol etil, } \\
\text { Bencil salicilato. } \\
\text { Polvo: Polietil metacrilato. Undecilenato } \\
\text { de Zinc }\end{array}$ \\
\hline $\begin{array}{l}\text { Material para rebasado resiliente } \\
\text { temporal a base de resina acrílica } \\
\text { (autocurado) }\end{array}$ & Dentusoft $\circledast$ & DMG Industria Argentina & $\begin{array}{l}\text { Líquido: Dibutil ftalato, alcohol desna- } \\
\text { turado } \\
\text { Polvo: Polietil metacrilato, peróxido } \\
\text { de benzoia, óxido de titánico }\end{array}$ \\
\hline $\begin{array}{l}\text { Material para rebasado resiliente } \\
\text { permanente a base de resina } \\
\text { acrílica (autocurado) }\end{array}$ & Dentuflex® & DMG Industria Argentina & $\begin{array}{l}\text { Líquido: N - Butil Metacrilato } \\
\text { Polvo: Polietil metacrilato, peróxido } \\
\text { de benzoíla. }\end{array}$ \\
\hline $\begin{array}{l}\text { Material para rebasado resiliente } \\
\text { temporal a base de resina acrílica } \\
\text { (termoplástico) }\end{array}$ & Dinabase ${ }^{\circledR}$ & $\begin{array}{l}\text { Quattro Ti Den Tech } \\
\text { Italia }\end{array}$ & $\begin{array}{l}\text { Líquido: Dispersantes } \\
\text { Polvo: Polímero vinil-acético } \\
\text { (monocomponente) }\end{array}$ \\
\hline
\end{tabular}

des recortados con una hoja de bisturí.

La prueba de dureza fue realizada en el período inicial y, después de 30 y 90 días de almacenamiento en agua destilada a $37^{\circ} \mathrm{C}$ con ayuda de un durómetro Shore A (modelo GSD 709 Teclock, Osaka, Japón) de acuerdo con las especificaciones D-2240 de la Sociedad Americana para Pruebas y Materiales (ASTM) ${ }^{20}$ a temperatura ambiente. Este método esta basado en la penetración de una aguja sobre la superficie del material con una carga constante de 10N. Los valores de dureza se expresaron en unidades Shore A, en una escala de 0 a 100, siendo la dureza proporcional a la penetración de la aguja, o sea, cuanto mayor la penetración, menor el valor indicado en la escala.

Para la prueba de rugosidad de superficie, fue utilizado un rugosímetro digital portátil modelo RP100 (Tonka Sul Americana Ltda, Amparo, São Paulo) con precisión de 0,01 $\mu \mathrm{m}$ y recorrido de medición de $6 \mathrm{~mm}$. Para cada muestra fueron realizadas 3 lecturas, (en dirección oblicua y transversal de derecha a izquierda y de arriba hacia abajo), que fueron transformadas en valores promedios, expresados en Ra (valor medio de la rugosidad superficial) ${ }^{8}$.

Los resultados obtenidos fueron sometidos al análisis de varianza seguido por el test de Tukey con 5\% de probabilidad. Para realizar el análisis estadístico fue utilizado el programa SANEST (Sistema de análisis estadístico).

\section{Resultados}

La tabla 2 muestra el análisis de varianza - Dureza shore A y la tabla 3 el análisis de varianza - Rugosidad los valores $F$.

De acuerdo con la tabla 4 se puede observar que los materiales Dentuflex, Coe-Soft, Dentusoft y Dinabase pre- sentaron, en este orden, los mayores valores medios iniciales de dureza Shore A. Además, después del periodo de almacenamiento ocurrió un aumento significativo en los valores de dureza en todos los materiales observados.

En la tabla 5 se observa que los materiales Dentuflex, Dentusoft, CoeSoft y Dinabase presentaron, en este orden, los mayores valores medios iniciales de rugosidad. Después del periodo de almacenamiento se observó que solamente el material Dentuflex presentó valores significativos menores de rugosidad, mientras que los otros materiales aumentaron significativamente.

\section{Discusión}

La eficacia de los materiales para rebasado blandos es atribuida a sus propiedades visco-elásticas y relacionadas principalmente con la flexibili- 


\begin{tabular}{|c|c|c|c|c|c|}
\hline \multicolumn{6}{|c|}{ Tabla e. Análisis de varianza - Dureza, Valor F } \\
\hline $\begin{array}{l}\text { Causas de } \\
\text { la variación }\end{array}$ & G.L & S.Q & Q.M & Valor $\mathrm{F}$ & Prob $>F$ \\
\hline Especifi & 1 & 1.0659671 & 1.0659671 & 3514.0785 & 0.00001 \\
\hline Material & 3 & 5.9866816 & 1.9955605 & 6578.5860 & 0.00001 \\
\hline Tempo & 2 & 1.46814444 & 0.7340722 & 2419.9502 & 0.00001 \\
\hline Esp*Mat & 3 & 0.02200696 & 0.0066899 & 22.0539 & 0.00001 \\
\hline Esp*Tem & 2 & 0.0555655 & 0.0277827 & 91.5889 & 0.00001 \\
\hline Mat*Tem & 6 & 0.1812155 & 0.0302026 & 99.5662 & 0.00001 \\
\hline Esp*Mat*Tem & 6 & 0.1019279 & 0.0169880 & 56.0027 & 0.00001 \\
\hline Resíduo & 96 & 0.0291208 & 0.0003033 & & \\
\hline Total & 119 & 8.9086924 & & & \\
\hline
\end{tabular}

\begin{tabular}{|lccccc|}
\hline \multicolumn{7}{|c|}{ Tabla 3. Análisis de varianZa - Rug0Sidad, Valor F } \\
\hline $\begin{array}{l}\text { Causas de } \\
\text { la variación }\end{array}$ & G.L & S.0 & Q.M & Valor F & Prob >F \\
\hline Material & 3 & 8.5887113 & 2.8629038 & 299.5977 & 0.00001 \\
\hline Tempo & 2 & 6.7939423 & 3.3969711 & 355.4869 & 0.00001 \\
\hline Mat*Tem & 6 & 29.3691661 & 4.8948610 & 512.2384 & 0.00001 \\
\hline Resíduo & 48 & 0.4586796 & 0.0095558 & & \\
\hline Total & $\mathbf{5 9}$ & $\mathbf{4 5 . 2 1 0 4 9 9 2}$ & & & \\
\hline Media general $=$ & 1.843167. & Coeficiente de varianza $=5.304 \%$ & & \\
\hline
\end{tabular}

\begin{tabular}{|c|c|c|c|c|c|c|}
\hline & Iabla 4.V & $\begin{array}{l}\text { ofes m } \\
\text { ci|al, d }\end{array}$ & $\begin{array}{l}\text { IS L des } \\
\text { ués de }\end{array}$ & gadron & व duгеzة: & \\
\hline Material & Inicial & $\begin{array}{l}\text { Desvío } \\
\text { Padrón }\end{array}$ & 30 días & $\begin{array}{l}\text { Desvío } \\
\text { Padrón }\end{array}$ & 90 días & Desvío \\
\hline Dentuflex & $68,2 \quad a, \Omega$ & $\pm 0,013$ & 85,2 b,$\Omega$ & $\pm 0,043$ & 87,8 b,$\Omega$ & $\pm 0,021$ \\
\hline Coe-Soft & 25,0 a, $\Psi$ & $\pm 0,007$ & $48,0 \quad b, \Psi$ & $\pm 0,017$ & 48,8 b, $\Psi$ & $\pm 0,010$ \\
\hline Dentusoft & $18,8 \quad a, \Phi$ & $\pm 0,010$ & 47,8 b, $\Psi$ & $\pm 0,017$ & $68,2 C, \Phi$ & $\pm 0,004$ \\
\hline Dinabase & $0,0 \quad a, \sum$ & $\pm 0,000$ & 28,2 b, $\Phi$ & $\pm 0,000$ & $28,4 \quad b, \sum$ & $\pm 0,005$ \\
\hline \multicolumn{7}{|c|}{$\begin{array}{l}\text { Los valores medios seguidos del mismo signo (en la columna) y letra minúscula (en la línea) no difie- } \\
\text { ren estadísticamente entre si, al } 5 \% \text { de probabilidad ( } p<0,05) \text {, por el Test de Tukey. } \\
\text { D.M.S. } \mathbf{5} \% 00.2878 \text { - D.M.S. } 1 \% 00.03513\end{array}$} \\
\hline
\end{tabular}

dad del material16,17; lo que mejora la absorción y redistribución de la energía generada por las fuerzas oclusales durante la función masticatoria. La manutención de esta propiedad constituye uno de los mayores problemas en la utilización de estos materiales, ya que la mayoría no es estable en un medio acuoso como la cavidad oral.

De acuerdo con Polyzois ${ }^{21}$ los valores de dureza de los materiales blandos varían de acuerdo con su composición química, lo que coincide con los resultados obtenidos en este estudio, en el cual hubo diferencias estadísticamente significativas entre los valores de dureza iniciales de los materiales evaluados. Inicialmente, Dentuflex presentó en todos los periodos, el mayor valor de dureza superficial comparado a los demás materiales. Esto puede estar relacionado, principalmente, con el hecho de estar clasificado por el fabricante como material permanente, conteniendo entonces una menor cantidad de plastificante, aproximándose a las características de la resina acrílica.

Así, el material Dinabase presentó dureza inicial cero, permaneciendo con los menores valores de dureza después del almacenamiento. A pesar de Dinabase ser un copolímero, es un material termoplástico monocomponente de alta viscosidad, absorbiendo poca agua del medio ambiente lo que hace que la característica de resilencia de este material permanezca por más tiempo.

Los valores iniciales de dureza de los materiales Coe-Soft y Dentusoft están de acuerdo con los resultados encontrados en la literatura ${ }^{22-25}$, siendo aceptable clínicamente ${ }^{26}$. Después del período de almacenamiento se 


\begin{tabular}{|c|c|c|c|c|c|c|}
\hline \multicolumn{7}{|c|}{$\begin{array}{l}\text { Tabla 5. Valores medios u desvío padrón de rugosidad: } \\
\text { inicial, después de } 30 \text { y g0 días }\end{array}$} \\
\hline Material & Inicial & $\begin{array}{l}\text { Desvío } \\
\text { Padrón }\end{array}$ & 30 días & $\begin{array}{l}\text { Desvío } \\
\text { Padrón }\end{array}$ & 90 días & Desvío \\
\hline Dentuflex & $2,304 \quad a, \sum$ & $\pm 0,0618$ & $1,628 \mathrm{~b}, \Psi$ & $\pm 0,1388$ & $1,424 \quad C, \Phi$ & $\pm 0,0577$ \\
\hline Coe-Soft & $1,240 \quad a, \Phi$ & $\pm 0,0857$ & $1,432 a, \Phi$ & $\pm 0,0909$ & 1,528 b, $\Phi$ & $\pm 0,0852$ \\
\hline Dentusoft & $1,610 \quad a, \Psi$ & $\pm 0,0509$ & $1,644 \quad a, \Psi$ & $\pm 0,0876$ & $1,972 \quad b, \Psi$ & $\pm 0,0634$ \\
\hline Dinabase & $0,344 \quad \mathrm{a}, \Omega$ & $\pm 0,0270$ & $3,320 \mathrm{~b}, \Omega$ & $\pm 0,1749$ & 3,672 C, $\Omega$ & $\pm 0,1404$ \\
\hline \multicolumn{7}{|c|}{$\begin{array}{l}\text { Los valores medios seguidos del mismo signo (en la columna) y letra minúscula (en la línea) no difie- } \\
\text { ren estadísticamente entre si, al } 5 \% \text { de probabilidad }(p<0,05) \text {, por el Test de Tukey. } \\
\text { D.M.S. } \mathbf{5} \%=\mathbf{0 . 1 6 4 8 1} \text { - D.M.S. } \mathbf{1} \%=\mathbf{0 . 2 0 3 7 2}\end{array}$} \\
\hline
\end{tabular}

verificó un aumento significativo en los valores de dureza de estos materiales (tabla 1), lo que puede estar relacionado a la pérdida del plastificante de bajo peso molecular asociado a la liberación de sub-productos como el alcohol etílico para el medio, al mismo tiempo que el agua es absorbida por el polímero ${ }^{17}$.

Además de la flexibilidad de estos materiales también es de gran relevancia evaluar la característica superficial de ellos ya que la presencia de rugosidades puede causas acumulaión de hongos y bacterias, llevando a la aparición de estomatitis. Por este motivo es ideal que las superficies de los materiales utilizados para la confección de prótesis sean lisas para minimizar o impedir el acumulo de placa bacteriana ${ }^{22}$, aunque que sea por un periodo de tiempo corto.

En nuestro estudio, las diferencias significantivas entre los valores iniciales de rugosidad de los materiales para rebasado están relacionados, probablemente, con la composición y consistencia de cada uno de ellos (tabla 4). Por ejemplo, para llenar los moldes con el material Dinabase fue necesario conferir cierta presión en el material, debido a que se presenta más viscoso. Por otro lado, los otros materiales poseen una consistencia regular, presentando mejor fluidez, consiguiendo copiar mejor los detalles de la superficie a ser reproducida. Esa superficie esta constituida por yeso especial presentando 1,2 Ra de rugosidad, valores cercanos a aquellos encontrados para los materiales Dentuflex, Dentusoft y Coe Soft, lo que les proporcionó mayores valores iniciales de rugosidad.

Después del período de almacenamiento, se verificó aumento en los valores de rugosidad en todos los materiales, con excepción del material Dentuflex (Tabla 4). De acuerdo con Massironi27, después de 3 a 4 semanas de uso, los materiales resilientes presentan pérdida de elasticidad, lo que torna a los materiales más rugosos, permitiendo retención de placa y desarrollo de microorganismos. Sin embargo, el material Dentuflex presentó valores de rugosidad menores después del almacenamiento y eso puede estar asociado a la mayor dureza de este material, pues para Frisch $^{28}$ cuanto mayor sea la dureza del material resiliente, mas lisa será la superficie.

De acuerdo con los resultados encontrados en este estudio, el tiempo de uso, según los fabricantes (Dentuflex - permanente, Coe-Soft y Dentusoft - de 1 a 3 meses y, Dinabase 1 mes), son clínicamente aceptables. Entre tanto, este estudio, por ser realizado «in vitro», presenta limitaciones, lo que hace necesario la realización de trabajos clínicos, para complementar los hallazgos de los estudios realizados en laboratorio.

\section{Conclusión}

Dentro de las limitaciones de este estudio, las siguientes conclusiones fueron observadas:

1. Los materiales estudiados presentaron entre si, diferentes valores iniciales de dureza Shore A y rugosidad, estadísticamente significativos.

2. El periodo de almacenamiento influenció significativamente en los valores de dureza Shore A y rugosidad de todos los materiales. 
Para profundizar en la lectura de este tema, el/los autor/es considera/an interesantes los artículos que aparecen señalados del siguiente modo: *de interés ${ }^{*}$ de especial interés.

1. Fujii K, Arikawa H, Kanie T, Ban S, Inoue M. Settings Characteristics of Silicone-based Resilient Denture Liners. Dent Mat J 2004;23:566-71.

2. Amin WM, Fletcher AM, Ritchie GM. The nature of the interface between polymethyl methacrylate denture base materials and soft lining materials. J Dent 1981;9:336-46.

3. Travaglini EA, Gibbons P, Craig RG. Resilient liners for dentures. J Prosthet Dent 1960; 10:664-72.

4. Sertgöz A, Kulak Y, Gedik H, Taskonak B. The effect of thermocycling on peel strength of six soft lining materials. J Oral Rehabil 2002;29: 583-87.

5. Bascom PW. Resilient denture base materials. J Prosthet Dent 1966;16:646-9.

6. Wilson HJ, Tomlin HR. Soft lining materials: some relevant properties and their determination. J Prosthet Dent. 1969;21:244-50.

$7 * *$. McCabe JF. Soft lining materials: composition and structure. J Oral Rehabil 1976;3:27378.

Analizó la composición de cinco materiales de rebase resilientes a base de resina acrílica. El autor concluyó que la resiliencia o módulo de elasticidad de los materiales con la misma composición química depende de la cantidad de plastificante y de la temperatura de transición vítrea del polímero.

8. Loney RW, Price RBT, Murphy DG. The Effect of Polishing on Surface Roughness of Tissue Conditioners. Int J Prosthodont 2000;13:20913.

9. Wilson HJ, McLean JW, Brown D. Materiais dentários e suas aplicações clínicas. São Paulo: Editora Santos,1989:101-9.

10. McCabe JF. A Polyvinylsiloxane denture soft lining material. J Dent 1998;26:521-6.

11. Casey DM, Scheer EC. Surface treatment of a temporary soft liner for increased longevity. J Prosthet Dent 1993;69:318-34.

12. Wright PS. Composition and Properties of Soft lining materials for acrylic dentures. J Dent 1981;9:210-23.

13. Jepson NJ, McCabe JF, Storer R. Evaluation of the viscoelastic properties of denture soft lining materials. J Dent 1993;21:163-70.

14. Braden M, Wright PS, Parker S. Soft lining materials-a review. Eur J Prosthodont Restor Dent 1995;3:163-74.

15. Wagner WC, Kawano F, Dootz ER, Koran A3rd. Dynamic viscoelastic properties of processed soft denture liners: part II-Effect of aging. J Prosthet Dent 1995;74:299-304.

16. Murata H,Taguchi N, Hamada T, Mccabe JF. Dynamic viscoelastic properties and the age changes of long-term soft denture liners. Biomaterials 2000;21:1421-27.

17. Murata H, Taguchi N, Hamada T, Kawamura M, Mccabe JF. Dynamic viscoelasticity of soft liners and masticatory function. J Dent Res 2002;81:123-28

18. Bulad K, Taylor RL, Verran J, McCord JF. Colonization and penetration of denture soft lining materials by Candida albicans. Dental Mater 2004;20:167-75.

19. Machado AL, Breeding LC, Puckett AD. Effect of microwave disinfection on the hardness and adhesion of two resilient liners. J Prosthet Dent 2005;94:183-9.

20. American society for testing and materials Standard test method for rubber propertyDurometer hardness. Annual book of ASTM standards rubber/ASTM-D 2240-81. 1983;8: 332-35.
21. Polyzois GL, Frangou MJ. Influence of curing method, sealer and water storage on the hardness of the soft lining material over time. J Prosthodont 2001;10:42-5.

22. Kazanji MNM, Watkinson AC. Soft lining materials: their absorption of, and solubility in, artificial saliva. Br Dent J 1988;165:91-4.

23. Qudah S, Hugget R, Harrison A. The effect of thermocycling on the hardness of soft lining materials. Quintessence Int 1991;22:575-80.

24. Dootz ER, Koran A, Craig RG. Comparison of the physical properties of 11 soft denture liners. J Prosthet Dent 1993;69:114-9.

25*. Yoeli Z, Miller V, Zeltser C. Consistency and softness of soft liners. J Prosthet Dent 1996; 75:412-8.

Se evaluó la dureza de cuatro materiales para rebasado resilientes autopolimerizables (Coe Soft, Flexacryl, Lyner e Permasoft) comparados con dos materiales de rebase resilientes a base de silicona (Molloplast-B e Permaflex). Hubo alteración de la dureza de todos los materiales con el tiempo, a excepción de los materiales resilientes a base de silicona, que permanecieron constantes. El valor de dureza varió significativamente con el espesor de la muestra.

26. Gonzalez JB. Use of Tissue Conditioners and Resilient Liners. Dent Clin North Am 1977;21: 249-59.

27. Massironi D. Mucosal conditioning in a postextraction and post-implantation mobile prosthesis: use of an innovative material. Disponible en: URL: htttp://www.dinabase.ch/scientific.html.

28. Frisch J, Levin MP, Bhasker SN. Clinical study of fungal growth on tissue conditioners. J Am Dent Assoc 1968;76:591-2. 